\title{
Médiévales
}

Langues, Textes, Histoire

56 | printemps 2009

Pratiques de l'écrit

\section{Les formulaires et la pratique de l'écrit dans les actes de la vie quotidienne ( $\mathrm{VI}^{\mathrm{e}}-\mathrm{X}^{\mathrm{e}}$ siècle)}

Formularies and the everyday use of the written word (6th-10th century).

\section{Alice Rio}

\section{OpenEdition}

\section{Journals}

Édition électronique

URL : https://journals.openedition.org/medievales/5525

DOI : 10.4000/medievales.5525

ISSN : 1777-5892

\section{Éditeur}

Presses universitaires de Vincennes

\section{Édition imprimée}

Date de publication : 30 juin 2009

Pagination : 11-22

ISBN : 978-2-84292-232-0

ISSN : 0751-2708

\section{Référence électronique}

Alice Rio, «Les formulaires et la pratique de l'écrit dans les actes de la vie quotidienne (vie-xe siècle) », Médiévales [En ligne], 56 | printemps 2009, mis en ligne le 21 septembre 2011, consulté le 22 avril 2022. URL : http://journals.openedition.org/medievales/5525; DOI : https://doi.org/10.4000/ medievales. 5525

Ce document a été généré automatiquement le 22 avril 2022.

Tous droits réservés 


\title{
Les formulaires et la pratique de l'écrit dans les actes de la vie quotidienne $\left(\mathrm{VI}^{\mathrm{e}}-\mathrm{X}^{\mathrm{e}}\right.$ siècle)
}

\author{
Formularies and the everyday use of the written word (6th-10th century).
}

\section{Alice Rio}

1 Le nombre de documents du haut Moyen Âge qui nous est parvenu est si faible que la question de l'usage de l'écrit pour cette période revient surtout à savoir jusqu'où extrapoler: dans quelle mesure les documents d'archives qui ont survécu sont-ils représentatifs de l'ensemble des transactions faites au cours de cette période, et quelle proportion de la population concernent-ils? La question de l'usage de l'écrit et de son rapport à la société est ainsi traitée de façons radicalement différentes selon le point de vue adopté par les historiens, mais les évaluations présentées par ceux-ci, qu'elles soient minimalistes ou maximalistes, dépassent rarement le stade de la profession de foi, précisément en raison d'un manque de preuves concrètes. Le but de cette contribution est de montrer qu'un certain type de source peut nous aider à évaluer la pratique de l'écrit plus précisément: les formulaires mérovingiens et carolingiens, souvent cités à ce propos mais, à l'exception notable des travaux d'Ian Wood et de Warren Brown, en général insuffisamment pris en compte ${ }^{1}$. Je voudrais ici examiner les recoupements et les différences entre ce corpus de formules et l'ensemble des documents d'archives originaux, et les points de vue distincts offerts par ces deux types de source sur la pratique de l'écrit dans les royaumes francs $\mathrm{du} \mathrm{VI}^{\mathrm{e}}$ au $\mathrm{x}^{\mathrm{e}}$ siècles.

La portée sociale des documents d'archives

2 Comme on le sait, toute évaluation de l'usage de l'écrit au cours de cette période est rendue difficile en raison de distorsions profondes dans la préservation des documents : ceux-ci ne survivent en général qu'à travers des archives ecclésiastiques, seules à posséder le niveau de continuité institutionnelle requis pour la préservation de documents dans le très long terme. Ces archives contiennent surtout des documents ayant trait aux droits sur la propriété foncière, puisque ceux-ci étaient les seuls à conserver leur utilité même des siècles après leur rédaction. La question est de savoir si 
cette distribution est représentative, c'est-à-dire si notre corpus documentaire reflète un déséquilibre réel dans l'usage de l'écrit entre les sphères laïques et ecclésiastiques, ou s'il est seulement le résultat des aléas de la conservation. Certains historiens, par exemple Michael Richter, ont conclu, en raison du petit nombre de manuscrits et de documents pouvant avec certitude être attribués à des laïcs, que ceux-ci n'utilisaient pas l'écrit et réglaient leurs affaires essentiellement par le biais de pratiques orales, et que les sources écrites en général ne représentent donc que le point de vue étroit d'institutions religieuses, seules à maintenir la tradition écrite romaine, et sans rapport avec les expériences de la grande majorité des laïcs ${ }^{2}$. Dans un sens, cette approche pourrait être considérée comme hyper-critique, mais elle l'est seulement à l'égard des sources écrites, se fondant par ailleurs sur des suppositions assez arbitraires, comme juger que l'usage de l'écrit est par principe opposé à l'identité «barbare ", même après la chute de l'Empire, ce qui revient à peu près à un raisonnement circulaire. Établir que les modes de communication orale étaient importants (et ils l'étaient sûrement) n'autorise pas à en conclure que l'écrit ne l'était pas.

Dans le camp opposé, Rosamond McKitterick, Janet Nelson et le groupe de recherche dit "Bucknell », à l'origine du livre The Settlement of Disputes in Early Medieval Europe, ont au contraire défendu l'idée de l'importance des formes écrites pour le gouvernement et l'administration des biens laïcs avant le $\mathrm{xII}^{\mathrm{e}}$ siècle, que Michael Clanchy avait désigné comme l'époque de la renaissance de l'usage de l'écrit, minimisant ainsi cet usage durant la période antérieure ${ }^{3}$. Un examen détaillé de plusieurs ensembles de documents d'archives, ainsi que des études sur la diffusion des lois et des capitulaires, ont joué un rôle important dans l'élaboration de cette perspective maximaliste. En particulier, l'étude de Rosamond McKitterick sur les archives de Saint-Gall, qui conservent un nombre exceptionnel de documents originaux, a démontré la présence, parmi des documents liés aux transactions de ce monastère, d'un certain nombre de chartes ayant trait à des laïcs, et de documents rédigés par des scribes laïcs indépendants ${ }^{4}$. Ces rares exemples sont étayés par des allusions fréquentes, dans les capitulaires et les notices de règlements de conflits, à l'utilisation de documents par des laïcs ${ }^{5}$. Cela indique que les intérêts des élites laïques étaient proches de ceux des élites ecclésiastiques : toutes deux en effet avaient comme objectif principal la préservation de leurs droits de propriété, pour laquelle l'usage de documents, en tant que source accrue d'autorité, présentait un avantage manifeste. Reste à savoir jusqu'à quel niveau dans l'échelle sociale cet usage se serait manifesté : Janet Nelson, en citant l'exemple des cartae que, d'après les capitulaires, les affranchis devaient porter sur eux, a avancé l'idée que l'usage de l'écrit était loin d'être restreint aux besoins des élites ${ }^{6}$.

4 Le fait que ces actes étaient non seulement écrits, mais de surcroît écrits en latin, pourrait évidemment constituer une objection majeure à cette interprétation maximaliste. La question de l'accessibilité de ces documents est donc essentielle pour étayer ou infirmer l'hypothèse d'une pratique de l'écrit largement répandue dans la société de cette époque. Janet Nelson a suggéré l'existence d'un type de literacy "passive" ou "pragmatique", limité à la reconnaissance de certains mots et expressions courants, et permettant ainsi même à des personnes n'étant pas membres de l'élite savante de comprendre le contenu essentiel d'un document ${ }^{7}$. Des recherches récentes ont également montré que la lecture directe ne constituait en fait qu'un moyen parmi d'autres d'accéder à ces textes, puisque ceux-ci étaient aussi lus à haute voix aux participants avant d'être confirmés. Selon cette perspective, l'oral et l'écrit auraient donc été complémentaires et interdépendants plutôt qu'opposés. La forme écrite 
constituerait ainsi une barrière potentielle moins importante qu'on ne l'a longtemps pensé: l'accessibilité de ces textes aurait dépendu non pas d'un haut degré d'alphabétisation, mais du niveau de langue utilisé et de la proportion de la population capable de le comprendre.

Le langage, comme l'écriture, est une forme de technologie, et par conséquent aussi un instrument de pouvoir. La question est de savoir si celui des documents de cette époque visait à la communication, s'il était censé être accessible à une large portion de la société, ou s'il visait à l'exclusion, c'est-à-dire s'il était au contraire destiné à renforcer les intérêts d'une élite qui aurait été la seule à le maîtriser'. Le langage des documents de cette époque a longtemps été considéré comme à la fois trop archaïque et trop décadent pour être un instrument efficace de communication : selon ce point de vue, le latin des documents du haut Moyen Âge était une langue morte, une langue d'érudits, accessible seulement à un petit nombre de scribes, qui eux-mêmes n'en avaient pas une connaissance très sûre ${ }^{10}$. Ces arguments ont été balayés par les recherches récentes de Roger Wright et surtout de Michel Banniard, qui, au lieu de se concentrer sur les " erreurs " et déviations grammaticales du latin du haut Moyen Âge par rapport au latin classique, ont démontré la coexistence de plusieurs niveaux de langue à caractères linguistiques distincts, allant du latin classicisant à des formes plus proches du protoroman. Depuis l'émergence de la sociolinguistique, le langage n'est plus considéré comme un monolithe que l'on maitrise ou non, mais comme un ensemble fluctuant selon l'usage, le registre et le niveau social de ceux qui le pratiquent ${ }^{11}$.

6 Cette approche plus nuancée peut expliquer le curieux mélange d'archaïsme et de renouvellement caractéristique $\mathrm{du}$ style documentaire de cette période: cette contradiction apparente vient du fait qu'à l'inverse des textes littéraires, ces documents n'étaient pas restreints à un seul niveau de communication, mais alternaient entre plusieurs niveaux. Les philologues des années 1950, dans le but d'étayer l'argument que le latin mérovingien était une langue savante, citaient surtout des exemples tirés de préambules, toujours caractérisés par un haut degré de formalisme, et écrits dans un style délibérément difficile, que les scribes eux-mêmes ne semblent pas toujours avoir compris: ces passages étaient destinés à prêter à la transaction décrite ce que Josef Herman a appelé « la dignité légalisante, sanctifiante de la tradition écrite $»^{12}$. Même si ces préambules n'étaient pas compris par la majorité des participants, cela n'avait guère de conséquence pour la compréhension du contenu essentiel de la transaction, puisque leur propos était limité le plus souvent à des remarques très générales. À l'inverse, les exposés et dispositifs formant le cœur de l'acte sont exprimés dans une langue beaucoup plus simple, indiquant le souci de faciliter leur compréhension : c'est surtout là qu'on trouve des formes proto-romanes, et aussi un usage plus fréquent de prépositions, clarifiant les fonctions grammaticales et compensant ainsi l'éventail réduit des cas. Les descriptions des limites de propriétés foncières étaient parfois écrites dans la langue vernaculaire, indiquant encore un désir d'inclusion important même dans les régions où celle-ci n'était pas issue du latin ${ }^{13}$. Ce souci de faciliter la communication des éléments essentiels semble avoir persisté à l'époque carolingienne: la renaissance carolingienne passe en effet à peu près inaperçue dans la langue des documents, n'affectant en fait que l'orthographe. Bien que la langue des documents soit loin de nous donner un reflet clair de la langue « populaire » de cette période, elle tendait visiblement à élargir la participation. 
$7 \quad$ Ni la forme écrite de ces documents ni leur usage du latin n'auraient donc constitué en principe un obstacle insurmontable à leur accessibilité à un grand nombre de personnes. Cependant, il faut bien avouer qu'établir l'absence d'obstacles n'équivaut pas pour autant à une preuve positive: malgré ses aspects séduisants, l'argument maximaliste souffre d'un manque de preuves concrètes. On en revient donc toujours à décider jusqu'à quel point il est légitime d'extrapoler à partir de rares exemples : en l'occurence, si les documents concernant des laïcs étaient vraiment l'exception, ou s'ils ne constituent au contraire que la partie émergée d'un iceberg.

L'apport des formulaires

8 Un autre type de source peut nous permettre d'évaluer plus précisément l'étendue et la portée des lacunes de notre corpus de documents d'archives, et étayer plus fermement l'argument maximaliste: les formulaires mérovingiens et carolingiens. Les formules sont des modèles de documents, le plus souvent rassemblés dans des collections, ou formulaires, et destinés à aider à la rédaction d'actes futurs. L'exemple le plus ancien date vraisemblablement de la fin du vi $\mathrm{v}^{\mathrm{e}}$ siècle, les plus tardifs du $\mathrm{x}^{\mathrm{e}}$. L'absence totale de collections de ce genre pour l'époque romaine pourrait mener à un argument selon lequel l'utilisation de formules indiquerait en soi un déclin dans l'usage de l'écrit, si l'on considère que seuls des scribes peu compétents, moins familiers de la rédaction, auraient eu besoin de suivre des modèles ${ }^{14}$. Mais l'existence de formes documentaires standardisées témoigne au contraire d'un usage de l'écrit fréquent: après tout, nos notaires actuels utilisent systématiquement des contrats standards, et on les accuse rarement d'analphabétisme ${ }^{15}$. La création de modèles de documents ne se justifie que si on en attend un gain de temps, donc dans une situation où la demande de documents est forte, et leur contenu répétitif : c'est seulement lorsque l'usage de documents n'est que rare ou même exceptionnel qu'on peut se permettre le luxe d'une rédaction particulière. Il ne faudrait d'ailleurs pas sous-estimer le niveau d'expertise requis pour la mise en oeuvre de ces modèles : la plupart ne pouvaient pas être réutilisés tels quels, et nécessitaient des modifications prenant en compte les circonstances à l'origine du nouveau document. L'existence de formulaires n'est donc pas le signe d'une pratique de l'écrit marginale, bien au contraire.

Bien que ces textes soient, à la fois par leur forme et leur contenu, très semblables aux documents d'archives, ils ne remplissaient pas la même fonction, et n'étaient pas préservés de la même manière, nous permettant ainsi d'accéder à un nouveau point de vue grâce à un ensemble de textes profondément différents. Les formulaires documentent avant tout les attentes des scribes, en nous montrant les modèles qu'ils jugeaient nécessaires de conserver pour l'avenir, et les situations qu'ils s'attendaient à rencontrer au cours de leur vie professionnelle. On peut voir dans les manuscrits que ces scribes modifiaient le plus souvent leurs collections de départ afin de les adapter à leurs propres besoins et attentes, en les réorganisant, en y ajoutant ou en enlevant des textes. Parce que les formules étaient conservées selon leur utilité pour la production de nouveaux documents au jour le jour - c'est-à-dire selon un principe de sélection radicalement étranger à celui des documents d'archives, qui ne survivaient que lorsque leur contenu présentait une utilité à long terme pour l'institution qui les conservait -, elles ne souffrent pas des mêmes distorsions. Elles traitent de sujets beaucoup plus variés : à côté de modèles de documents ayant trait aux transactions foncières d'églises ou de monastères, tels qu'on en trouve dans les archives, elles contiennent également des modèles de documents à valeur plus éphémère, documents que ces institutions ne 
jugeaient pas utile de préserver, et dont on ne trouve donc aucune trace dans leurs archives. Les formulaires comprennent ainsi des exemples de documents ayant trait à des résolutions de conflits concernant des meurtres, vols ou enlèvements, prêts, entrées en service, lettres de recommandation, qui auraient perdu toute valeur au plus tard à la mort des participants ${ }^{16}$.

10 Comme les chartes, les formules qui nous sont parvenues ont surtout été transmises par les institutions ecclésiastiques. Bien qu'il soit tout à fait possible, et même probable, que des scribes laïcs aient aussi utilisé des collections de ce genre, aucune ne semble avoir survécu. Malgré cela, nos formulaires contiennent un grand nombre, même une majorité, de modèles de documents ne concernant que des laïcs, qui semblent s'être souvent adressés aux scribes d'institutions religieuses pour la rédaction de leurs actes : bien que ces institutions aient rarement préservé ce genre de document, leurs scribes devaient néanmoins se préparer à les écrire, ce qui explique leur présence dans leurs collections de modèles ${ }^{17}$. À l'inverse des documents d'archives, les formules nous montrent des églises et des monastères qui ne servaient pas seulement leurs propres intérêts, mais aussi ceux des communautés laïques dans leur environnement. L'origine ecclésiastique des formulaires ne les empêche donc pas d'être représentatifs du milieu laïc. Ces communautés laïques semblent avoir été activement impliquées dans la rédaction de ces documents, et en avoir tiré parti dans leur propre intérêt. On trouve ainsi des monastères conservant et recopiant jusqu'au $\mathrm{IX}^{\mathrm{e}}$ siècle des modèles d'actes de divorce par consentement mutuel : bien qu'ils aient forcément dû désapprouver cette pratique (même si la législation canonique ne s'intéressait pas encore de très près à ce sujet $\left.^{18}\right)$, ils devaient néanmoins se plier aux exigences de la population laïque vivant dans leur zone d'influence. La présence dans les formulaires de ce type de modèles reflète donc une réelle demande, de la part de la société laïque, de documents écrits.

11 Les formulaires nous permettent d'observer des laïcs utilisant des documents écrits de manière comparable aux fondations religieuses, et avec une compréhension de leur fonctionnement tout aussi sophistiquée : on y trouve ainsi un couple avertissant le lecteur à l'avance que son testament comprendrait de nombreuses ratures et corrections, puisqu'il se réservait la possibilité de modifier ce testament dans l'avenir, et que cela ne devait pas entraîner de doute sur sa validité ${ }^{19}$, montrant qu'il était conscient, tout en tirant un parti actif du pouvoir légitimant de l'écrit, de la nécessité de se garder des pièges qui lui étaient propres. Ce texte, comme beaucoup d'autres, semble indiquer que les laïcs conservaient leurs documents chez eux. Plusieurs formulaires contiennent des modèles à suivre afin de remplacer des documents détruits ou perdus : cette procédure comprenait un rapport signé par des témoins et voisins confirmant les circonstances de la perte de ces documents, ainsi qu'une liste des droits que ceux-ci avaient contenus; ce témoignage devait ensuite être présenté devant un tribunal, qui produisait un document de remplacement (appennis), lequel devait apparemment être affiché en public pendant trois jours avant de pouvoir être sanctionné20. Un de ces modèles décrit un laïc qui, à la suite d'une attaque, avait perdu tous les documents qu'il gardait dans sa maison : la formule nous dit que ces documents comprenaient des actes de vente, de prêt, de donation, de douaire, des notices de jugement et de résolution de conflit, des contrats, des échanges, « et beaucoup d'autres choses en trop grand nombre pour pouvoir les énumérer en détail», et qu'ils témoignaient que de nombreuses terres lui avaient été vendues ${ }^{21}$. Les laïcs pouvaient ainsi posséder un grand nombre de documents liés à des sujets très divers, et constituant peut-être des archives de taille importante, bien que n'ayant guère de 
chances de survie dans le long terme. La perte d'un document pouvait visiblement entraîner une perte réelle et matérielle, comme dans le cas d'un couple qui, après avoir perdu le document par lequel un homme était entré dans son service en échange d'une somme d'argent, se trouva forcé d'abandonner tous ses droits sur sa personne ${ }^{22}$. Les laïcs, comme les églises, avaient donc un intérêt évident à faire confirmer leurs transactions par l'écrit, à les conserver, et à les faire remplacer en cas de perte. La question du milieu social de ces laïcs est importante. On en vient là à un autre trait frappant des formules par rapport aux documents d'archives: non seulement elles contiennent de nombreux exemples de documents à l'usage de laïcs, mais ces laïcs n'appartiennent pas nécessairement à des milieux sociaux très élevés. À côté de modèles pour des actes de vente ou de donation de villas, on trouve ainsi des exemples de transactions beaucoup plus modestes: par exemple des ventes, échanges ou baux concernant un seul champ ou une seule vigne, et décrivant souvent l'étendue et les limites de ces terrains très en détail ${ }^{23}$. On trouve aussi des modèles montrant que l'écrit était utilisé pour des résolutions de conflits relativement mineurs: le formulaire d'Angers nous présente ainsi deux documents rédigés à la suite du vol d'une seule jument ( $n^{\circ} 11$ et 13), et un autre concernant un vol de peaux de bétail $\left(n^{\circ} 24\right)$, ressemblant beaucoup à ceux qui concernent des meurtres ou des conflits liés à la propriété foncière. Une même culture documentaire pouvait donc s'appliquer à un grand nombre de cas, quelle que fût l'importance de l'objet du litige. Des collections telles que le formulaire d'Angers, qui ne contient que des transactions et règlements de conflits menés à l'échelle locale, montrent que les élites religieuses ou laïques n'étaient pas seules à savoir tirer parti de la sécurité que conférait la possession d'un document. Cela est d'ailleurs confirmé par le fait que, dans les conflits contenus dans ce formulaire, les élites ne semblent pas avoir gagné systématiquement: le formulaire d'Angers $\left(n^{\circ} 10\right)$ nous montre ainsi un individu défendant avec succès son statut d'homme libre devant un tribunal après avoir été accusé par quelqu'un qui se disait son maître.

13 Un examen du latin des formulaires confirme aussi le souci d'accessibilité déjà visible dans les documents d'archives de cette époque ${ }^{24}$. Comme les chartes, les formules présentent un usage restreint des cas, et un usage plus répandu des prépositions et des formes analytiques proches des langues romanes. Mais la langue des formules permet des comparaisons plus développées et nuancées : les formulaires rassemblent en effet plusieurs documents associés à un même scribe, qui, même s'il n'en était pas l'auteur, les a néamoins retravaillés, nous donnant ainsi un échantillon beaucoup plus large de choix de niveaux de langue. Marculf, par exemple, utilise beaucoup plus de prépositions dans son livre II, qui contient des modèles d'actes privés, que dans son livre I, qui contient des exemples de diplômes royaux : il n'y a ainsi que trois exemples du remplacement du génitif de la première déclinaison par de + - a dans le livre I, mais la même construction n'apparait pas moins de 28 fois dans le livre II. La forme analytique $a d+-a$ ou - am à la place d'un datif de la première déclinaison n'apparaît que sept fois dans le livre I, mais 44 fois dans le livre $\mathrm{II}^{25}$. Marculf utilise donc régulièrement des formes proto-romanes pour des modèles de documents privés, mais les évite pour les diplômes royaux, plus solennels et élaborés. Son recours à des formes grammaticales dites "vulgaires ", plus proches de la langue orale, n'était donc pas dû à un manque de maîtrise du latin, ou à un glissement linguistique inconscient ou incontrôlé, mais était le fruit d'un vrai choix: il adaptait son style à son public, maintenant un difficile équilibre entre le besoin de conserver une atmosphère 
traditionnelle de solennité et de formalisme, afin de donner du poids aux évènements décrits, et celui de créer un texte en pratique compréhensible pour les participants.

Les formulaires permettent donc de revoir à la hausse notre évaluation de la pratique de l'écrit au cours de cette période, et celle des lacunes de notre corpus de documents d'archives : en nous montrant comment les scribes concevaient leur travail, ils nous donnent, sinon une vue complète de l'iceberg, du moins une meilleure idée de sa taille et de sa forme. Bien que l'écrit, comme toute institution garantissant les droits de propriété privée, ait existé surtout pour servir les intérêts des élites, une plus grande proportion de la population semble néanmoins avoir pu en tirer parti : ces textes nous montrent que la pratique de l'écrit à cette époque était adaptable, moins exclusive qu'on ne s'y attendrait, et témoignent d'un usage répandu, au quotidien et à l'échelon local.

\section{NOTES}

1..I.N. wooD, « Administration, law and culture in Merovingian Gaul », dans

R. MCKITTERICK dir., The Uses of Literacy in Early Medieval Europe, Cambridge, 1990,

p. 63-81; W. BROWN, « When documents are destroyed or lost : lay people and archives in the early middle ages ", Early Medieval Europe, t. 11, 2002, p. 337-366. Cet article est fondé sur le premier chapitre de mon livre sur la question des formulaires, Legal Practice and the Written Word in the Early Middle Ages : Frankish Formulae, c. 500-1000, Cambridge, 2009. Je remercie vivement Jinty Nelson, Michel Banniard et Rosamond McKitterick d'avoir bien voulu lire et commenter la version anglaise de ce texte. 2.M. RICHTER, «"Quisquis scit scribere, nullum potat abere labore". Zur Laienschriftlichkeit im 8. Jahrhundert ", dans J. JARNUT, U. NONN et M. RICHTER dir., Karl Martell in seiner Zeit, Sigmaringen, 1994, p. 393-404 ; M. RICHTER, The Formation of the Medieval West : Studies in the Oral Culture of the Barbarians, Dublin, 1994: « [written sources were] of rather marginal importance to much of early medieval life, the output of the expertise of some individuals and groups possessed of no great social prestige » (p. VIII) ; « writing was throughout the early medieval centuries the domain of a small circle of specialists for rather narrowly circumscribed purposes... » (p. 262). Pour un argument similaire, voir aussi F.L. CHEYETTE, « The invention of the state », dans B.K. LACKNER et K.R. PHILIP dir., Essays in Medieval Civilization: The Walter Prescott Webb Memorial Lectures, Austin, 1979, p. 143-176, p. 149-156. Pour une critique de Richter, voir par exemple M. INNES, " Memory, orality and literacy in an early medieval society ", Past and Present, t. 158, 1998, 3-36, p. 7-8 ; M. INNES, State and Society in the Early Middle Ages : the Middle Rhine Valley (400-1000), Cambridge, 2000, p. 111-112 et 117-118.

3..R. MCKITTERICK, The Carolingians and the Written Word, Cambridge, 1989 ; R. MCKITTERICK dir., The Uses of Literacy in Early Medieval Europe. Dans ce volume, voir en particulier I.N. wooD, « Administration, law and culture in Merovingian Gaul », et J.L. NELSON, « Literacy in Carolingian government », p. 258-296. Pour le groupe « Bucknell », voir W. DAVIES et P. FOURACRE dir., The Settlement of Disputes in Early Medieval Europe, Cambridge, 1986, et 
Property and Power in Early Medieval Europe, Cambridge, 1995. M. CLANCHY, From Memory to Written Record, $2^{\mathrm{e}}$ édition, Oxford, 1993. Sur la pratique de l'écrit par les élites laïques, voir aussi P. WORMALD et J.L. NELSON dir., Lay Intellectuals in the Carolingian World, Cambridge, 2007.

4.R. MCKITTERICK, The Carolingians and the Written Word, op. cit., p. 77-134; sur une section de l'archive de St-Gall considérée comme ayant appartenu à un laïc, voir K. BULLIMORE, « Folcwin of Rankweil : the world of a Carolingian local official », Early Medieval Europe, t. 13, 2005, p. 43-77 ; P. ERHART et J. KLEINDINST, Urkundenlandschaft Rätien, Vienne, 2004 (Forschungen zur Geschichte des Mittelalters 7, Österreichische Akademie der Wissenschaften phil.-hist. Klasse, Denkschriften 319), p. 28-30, 83-90 et 167-219 $\left(\mathrm{n}^{\circ}\right.$ 11-37).

5.J. NELSON, « Literacy in Carolingian government », loc. cit. ; W. DAVIES et P. FOURACRE, "Conclusion », dans W. DAVIES et P. FOURACRE dir., The Settlement of Disputes in Early Medieval Europe, loc. cit., p. 207-240.

6..J. NELSON, « Literacy in Carolingian government », loc. cit., p. 262 ; cité par P.J. GEARY, «Land, language and memory in Europe, 700-1100 », Transactions of the Royal Historical Society, $6^{\text {e }}$ série, t. 9, 1999, p. 169-184 (p. 169).

7..J. NELSON, «Literacy in Carolingian government », loc. cit., p. 269-270. Sur l'idée de " pragmatic literacy », voir M.B. PARKES, « The literacy of the laity », dans D. DAICHES et A. THORLBY dir., Literature and Western Civilization : The Mediaeval World, Londres, 1973, p. 555-577 ; M. CLANCHY, From Memory to Written Record, op. cit., p. 236 et 247.

8..M. INNES, State and Society, loc. cit., p. 118 : « The ability to read, still less to write, a charter did not need to be widespread for the written word to play a central role in legal practice. » J. NELSON, « Literacy in Carolingian government », loc. cit., p. 266-267 ; R. MCKITTERICK, The Uses of Literacy, op. cit., p. 320-321 ; M. INNES, « Memory, orality and literacy », op. cit., surtout p. 3-10 et p.35-36 ; P. GEARY, " Land, language and memory ", loc. cit. Voir aussi B. Ітоск, Listening for the Text : On the Uses of the Past, Philadelphie, 1996, p. 5-6 ; R. FINNEGAN, Literacy and Orality: Studies in the Technology of Communication, Oxford, 1988.

9..Voir M. DE JONG, « Some reflections on mandarin language », dans E. CHRYSOS et I.N. wOoD dir., East and West : Modes of Communication. Proceedings of the First Plenary Conference at Merida, Leyde, 1999, p. 61-69.

10.D. NORBERG, Manuel pratique de latin médiéval, Paris, 1968, p. 29-31. Pour un argument similaire, voir aussi E. LÖFSTEDT, Late Latin, Oslo, 1959 ; C. MOHRMANN, Latin vulgaire, latin des chrétiens, latin médiéval, Paris, 1956 ; C. MOHRMANN, « Le latin prétendu vulgaire et l'origine des langues romanes », dans Centre de Philologie Romane, Strasbourg, 1961, p. 90-98 ; J. VIELLIARD, Le latin des diplômes royaux et des chartes privées de l'époque mérovingienne, Paris, 1927 ; R. FALKOWSKI, « Studien zur Sprache der Merowingerdiplome ", Archiv für Diplomatik, t. 17, 1971, p. 1-125. Pierre Riché contribua beaucoup à la dissémination de ce point de vue parmi les historiens (P. RICHÉ, Éducation et culture dans l'Occident barbare, $\mathrm{VI}^{e}$-VII ${ }^{e}$ siècles, Paris, 1962, p. 284-285).

11.R. WRIGHT, Late Latin and Early Romance in Spain and Carolingian France, Liverpool, 1982 ; R. WRIGHT dir., Latin and the Romance Languages in the Early Middle Ages, Londres/ New York, 1991 ; et les articles inclus dans R. WRIGHT, A Sociophilological Study of Late Latin, Turnhout, 2002 ; M. BANNIARD, Viva voce : communication écrite et communication orale du IV $V^{e}$ au IX siècle en Occident latin, Paris, 1992 ; M. BANNIARD, " Niveaux de langue et 
communication latinophone ", dans Comunicare e significare nell'alto medioevo, Spoleto, 2005 (Settimana internazionale di Studio 52), p. 155-208 ; M. BANNIARD, « Diasystèmes et diachronie langagière du latin parlé tardif au protofrançais », dans J. HERMAN et L. MONDIN dir., La transizione dal latino alle lingue romanze. Atti della tavola rotonda di linguistica storica. Università Ca'Foscari di Venezia, 14-15 giugno 1996, Tübingen, 1998, p. 131-153 ; M. BANNIARD, « Seuils et frontières langagières dans la Francia romane du VIII ${ }^{e}$ siècle ", dans JARNUT, NONN et RICHTER dir., Karl Martell in seiner Zeit, p. 171-191 ; M. BANNIARD, «Le latin mérovingien, état de la question », dans M. GOULLET et M. PARISSE dir., Les historiens et le latin médiéval, Paris, 2000, p. 17-30. Je remercie beaucoup Michel Banniard d'avoir bien voulu m'envoyer certains de ces articles.

12.J. HERMAN, « Sur quelques aspects du latin mérovingien : langue écrite et langue parlée ", dans M. ILIESCU et W. MARXGUT dir., Latin vulgaire - Latin tardif III. Actes du troisième colloque international sur le latin vulgaire et tardif (Innsbruck, 2-5 septembre 1991), Tübingen, 1992, p. 173-186 (p. 177).

13..P. GEARY, « Land, language and memory », loc. cit., p. 175-184. C'est aussi le cas pour les documents anglo-saxons.

14..I.N. wooD, « Administration, law and culture in Merovingian Gaul », loc. cit., p. 64 ; P. HEATHER, « Literacy and power in the migration period », dans A. BOWMAN et G. WOOLF dir., Literacy and Power in the Ancient World, Cambridge, 1994, p. 177-197 (p. 192-193). 15.Voir M. CLANCHY, From Memory to Written Record, loc. cit., p. 31, pour les royaumes anglo-saxons ; voir aussi W. DAVIES et P. FOURACRE, «Conclusion », loc. cit., p. 212. Henri Pirenne considérait déjà les formulaires comme le signe d'une pratique de l'écrit largement répandue (H. Pirenne, Mahomet et Charlemagne, Paris, 1937, p. 170-171). 16..P. GEARY, « Land, language and memory », loc. cit., p. 170.

17..Voir R. MCKITTERICK, The Carolingians and the Written Word, op. cit., p. 77-134; M. INNES, State and Society, op. cit., p. 111-118. Pour des exemples comparables en Bretagne et en Espagne, voir W. DAVIES, Small Worlds : The Village Community in Early Medieval Brittany, Londres, 1988, p. 137 - 38 ; W. DAVIES, Acts of Giving : Individual, Community, and Church in Tenth-Century Christian Spain, Oxford, 2007, p. 97.

18..Pour des exemples de divorce dans les formulaires, voir Formulae Andecavenses $n^{\circ} 57$, Formulae Marculfi II, 30, Formulae Turonenses $n^{\circ} 19$, Cartae Senonicae $\mathrm{n}^{\circ} 47$ et Formulae Salicae Merkelianae $\mathrm{n}^{\circ}$ 18, dans Formulae Merowingici et Karolini aevi, K. ZEUMER éd., Hanovre, 1886 (MGH Leges V). D. D'AVRAY, Medieval Marriage : Symbolism and Society, Oxford, 2005, p. 74-81; J.-A. MCNAMARA et S.F. WEMPLE, « Marriage and divorce in the Frankish kingdom », dans S.M. STUARD dir., Women in Medieval Society, Philadelphie, 1976, p. 96-124 ; A. RIO, « Formulae, legal practice and the settlement of disputes in the Frankish kingdoms : the formulary of Angers ", dans P. ANDERSON, M. MÜNSTER-SWENDSEN et H. voGT dir., Law before Gratian. Proceedings of the Third Carlsberg Academy Conference on Medieval Legal History, Copenhague, 2007, p. 21-34 (p. 26-27).

19.Formulae Marculfi II, 17.

20. Voir Formulae Andecavenses nos. 31-34, Formulae Arvernenses n ${ }^{\circ}$ 1, Formulae Marculfi I, 33-34, Formulae Turonenses $\mathrm{n}^{\circ \mathrm{s}}$ 27-28 et Add. $\mathrm{n}^{\circ}$ 7, et Cartae Senonicae $\mathrm{n}^{\circ \mathrm{s}} 38$ et 46 (dans Formulae Merowingici et Karolini aevi, K. ZEUMER éd., p. 14-15, 28, 63-65, 150-51, 162, 202 et 205-206). Cette procédure a fait l'objet de plusieurs études : K. ZEUMER, « Über den Ersatz verlorener Urkunden im fränkischen Reiche », Zeitschrift der Savigny-Stiftung für Rechtsgeschichte, Germanistische Abteilung, t. 1, 1880, p. 89-123 ; L. GOBIN, « Notes et documents concernant l'histoire d'Auvergne. Sur un point particulier de la procédure 
mérovingienne applicable à l'Auvergne : "l'institution d'apennis" ", Bulletin historique et scientifique de l'Auvergne, 1894, p. 145-153 ; plus récemment, voir aussi C. LAURANSONROSAZ et A. JEANNIN, « La résolution des litiges en justice durant le haut Moyen Âge : l'exemple de l'apennis à travers les formules, notamment celles d'Auvergne et d'Angers ", dans Le règlement des conflits au Moyen Âge, XXXI Congrès de la SHMES (Angers, juin 2000), Paris, 2001, p. 21-33 ; et, pour une approche différente, BROWN, « When documents are destroyed or lost : lay people and archives in the early middle ages ». 21..Formulae Andecavenses $\mathrm{n}^{\circ} 32$.

22..Formulae Andecavenses $\mathrm{n}^{\circ} 17$.

23.Voir, par exemple, Formulae Andecavenses no. 8, 21 et 22 ; Formulae Marculfi II, 20, 21 et 24 .

24.Pour des études spécialisées, voir J. PIRSON, « Le latin des formules mérovingiennes et carolingiennes », Romanische Forschungen, t. 26, 1909, p. 837-944 ; L. BESZARD, La langue des formules de Sens, Paris, 1910 ; A. UDDHOLM, Formulae Marculfi : Études sur la langue et le style, Uppsala, 1953.

25.Ce déséquilibre n'est pas noté par Sas dans son étude exemplaire sur la langue des formulaires d'Angers et de Marculf (L.F. SAS, The Noun Declension System in the Merovingian Period, New York, 1937).

\section{RÉSUMÉS}

Il est difficile de juger de la portée de l'usage de l'écrit au haut Moyen Âge parce que les transactions menées au bas de l'échelle sociale n'ont guère laissé de traces dans nos sources. Plusieurs historiens ont avancé l'hypothèse que l'usage de l'écrit était plus répandu qu'on ne pourrait s'y attendre à partir des documents d'archives qui nous sont parvenus, mais les limites imposées par les conditions de transmission des actes de la pratique rendent cette hypothèse pratiquement impossible à démontrer. Les formulaires, ou collections de modèles de documents, peuvent nous permettre d'évaluer plus précisément l'étendue des lacunes de ce corpus documentaire. Parce qu'ils étaient préservés en raison de leur utilité au jour le jour, et non selon leur capacité à protéger les intérêts d'établissements religieux dans le long terme, ils nous donnent une image plus représentative du genre de documents que les scribes s'attendaient à devoir écrire au cours de leur carrière, et constituent une source d'information importante sur l'usage de l'écrit par les laïcs, y compris ceux provenant de milieux sociaux moins élevés.

Formularies and the everyday use of the written word (6th-10th century). Judging the social impact of the use of the written word in the early middle ages is made difficult due to the dearth of surviving sources concerning transactions made by lower-status people. Several historians have put forward arguments that the written word was used more widely than the surviving archival evidence suggests, but the limits imposed by the conditions of transmission of documents make it almost impossible to be certain of this. Formularies, collections of models, offer a chance to gauge some of the gaps in this surviving archival evidence. Because they were preserved according to their day-to-day usefulness in drawing up new documents rather than according to the long-term interests of religious institutions, they give a more balanced view of what documents scribes expected they would need to write in the course of their career, and 
constitute important evidence for the use of the written word by lay people from a wide range of social backgrounds.

INDEX

Keywords : archives, literacy, formularies, written word, post-classical Latin

Mots-clés : formulaires, usage de l'écrit, latin post-classique

\section{AUTEUR}

ALICE RIO

Sidney Sussex College - Sidney Street - UK-Cambridge CB2 3HU 